\title{
Richtigkeit und Präzision bei verschiedenen Methoden zum Nachweis von Fructose im Sperma
}

\author{
Von G. Peter und V. Hauenstein \\ Aus der Biochemischen Abteilung (Leiter: Priv. Doz. Dr. G. Peter) der Dermatologischen Universitätsklinile \\ Würzburg
}

(Eingegangen am 28. Juni/28. August 1972)

Getestet wurden die Reaktionen der Fructose mit Anthron, Anthron + Thioharnstoff, Diphenylamin, Resorcin und die enzymatische Methode. Über die Bestimmung der Richtigkeit und Präzision mit den genannten Methoden beim Fructose-Nachweis im Sperma wird berichtet. Die statistische Analyse ergab, daß die beiden Anthron-Reagenzien für den Fructose-Nachweis wegen der zu großen Abweichung der Mittelwerte vom Sollwert $( \pm 25 \%)$ nicht zu empfehlen sind. Die Regressions- und Varianzanalyse ergaben, daß die Diphenylamin-, Resorcin- und enzymatische Methode einander ebenbürtig und gleich gut sind. Die Problematik und Aussagekraft der einzelnen Verfahren wird diskutiert.

\section{The accuracy and precision of different methods for the determination of fructose in spermatazoa}

Fructose was determined by reaction with anthrone, anthrone + thiourea, diphenylamine, or resorcinol, and by the enzymic method. The accuracy and precision of these methods are reported for the determination of fructose in spermatazoa. Statistical analysis showed that both the anthrone reagents are unsuitable for the determination of fructose, since they gave average values which varied from the theoretical by $+25 \%$. Regression and variance analyses showed that the diphenylamine, the resorcinol and the enzymic methods are all equally suitable. The associated problems and the diagnostic value of each method are discussed.

Bei andrologischen Untersuchungen wird der FructoseBestimmung bekanntlich eine große Bedeutung beigemessen (1). Einige von den in der Literatur (2) beschriebenen Verfahren werden in überwiegendem Maße auf breiter Ebene angewandt. Es bestehen aber recht unterschiedliche Meinungen über die Genauigkeit der einzelnen Methoden.

Wir haben daher den Versuch unternommen, die gängigsten Methoden auf ihren Aussagewert hin zu überprüfen. Im einzelnen wurde die Reaktion der Fructose mit Anthron (3), Anthron + Thioharnstoff (4), Diphenylamin (5), Resorcin (6) und die enzymatische Methode (7) getestet.

Diese vorwiegend für den Fructose-Nachweis in Harn und Blut benutzten Verfahren wurden für die SpermaMatrix teilweise modifiziert.

\section{Material und Methoden}

\section{Sperma}

Es wurde jeweils frisch gewonnenes Ejakulat von etwa 100 Versuchspersonen verwendet und sofort für die Analyse eingesetzt.

Standard-Fructoselösung

Die Konzentration der Stammlösung betrug $200 \mathrm{mg} / 100 \mathrm{ml}$ und wurde für die Aufstellung von Eichkurven entsprechend verdünnt.

Chemische Fructose-Bestimmung

Verfabren 1 (Diphenylamin)

$0,1 \mathrm{ml}$ Probe wurde mit $8 \cdot \mathrm{ml} \mathrm{CdSO}_{4}\left(13 \mathrm{~g} \mathrm{CdSO}_{4}+63,5 \mathrm{ml} 0,5 \mathrm{~mol} / 1\right.$ $\mathrm{H}_{2} \mathrm{SO}_{4}$ ad $1000 \mathrm{ml}$ ) gemischt, $1 \mathrm{ml} 1 \mathrm{~mol} / 1 \mathrm{NaOH}$ zugesetzt, gemischt, $10 \mathrm{~min}$ zentrifugiert $(3000 \mathrm{U} / \mathrm{min})$ und durch ein Faltenfilter filtriert. $4 \mathrm{ml}$ Filtrat wurden mit $2 \mathrm{ml} \mathrm{HCl}(\mathrm{d}=1,19)$ und $0,4 \mathrm{ml}$ Diphenylaminlösung ( $10 \mathrm{~g}$ Diphenylamin in $100 \mathrm{ml}$ abs.
Äthanol) gemischt, die Reagenzgläser locker verschlossen, $15 \mathrm{~min}$ in siedendem Wasserbad erhitzt, 3 min unter fließendem Wasser gekühlt, $4 \mathrm{ml} n$-Propanol zugesetzt, $20 \mathrm{~min}$ bei Raumtemperatur stehen gelassen und bei $620 \mathrm{~nm}$ die Extinktion bei $0,5 \mathrm{~cm}$ Schichtdicke gegen Wasser bestimmt. Der aus der Eichkurve abgelesene Wert wurde mit dem Faktor 91 multipliziert. Angabe in $\mu \mathrm{g} / \mathrm{ml}$ Sperma.

\section{Verfabren 2 (Resorcin)}

$0,1 \mathrm{ml}$ Probe wurde mit $0,3 \mathrm{ml}$ dest. Wasser gemischt, $2 \mathrm{ml}$ 10proz. $\mathrm{ZnSO}_{4}$-Lösung und $2 \mathrm{ml} 0,1 \mathrm{~mol} / 1 \mathrm{NaOH}$ zugegeben, 2 min in siedendem Wasserbad erhitzt, abgekühlt und durch ein Faltenfilter filtriert. $2 \mathrm{ml}$ des klaren Filtrats wurden mit $2 \mathrm{ml}$ Resorcinlösung $(0,1 \%$ in $96 \%$ Äthanol) und $6 \mathrm{ml} \mathrm{30proz.} \mathrm{HCl}$ versetzt, $8 \mathrm{~min}$ in einem Wasserbad von $80^{\circ} \mathrm{C}$ erhitzt, abgekühlt und die Extinktion bei $490 \mathrm{~nm}(1 \mathrm{~cm}$ Schichtdicke) abgelesen. Der aus der Eichkurve abgelesene Wert wurde mit 44 multipliziert. Angabe in $\mu \mathrm{g} / \mathrm{ml}$ Sperma. Variante (ohne Verwendung einer Eichkurve): Berechnung exfolgte nach $\frac{E_{\text {Pobe }}}{E_{\text {Standard }}} \cdot 2000$.

Verfabren 3 (Antbron)

$0,1 \mathrm{ml}$ Sperma wurde mit $4,9 \mathrm{ml} \mathrm{3proz} . \mathrm{HClO}_{4}$ enteiveißt und filtriert. $1 \mathrm{ml}$ des Filtrats wurde mit $2 \mathrm{ml}$ Anthronlösung $(0,2 \%$ in 96 proz. $\mathrm{H}_{2} \mathrm{SO}_{4}$ ) im Eisbad versetzt, weitere $30 \mathrm{~min}$ gekühlt und anschließend bei $620 \mathrm{~nm}$ die Extinktion bei $0,5 \mathrm{~cm}$ Schichtdicke abgelesen. Das Extinktionsverhältnis von Probe: Standard multipliziert mit 2000 ergibt die Konzentration in $\mu \mathrm{g} / \mathrm{ml} \mathrm{Sperma.}$

\section{Verfabren 4 (Antbron + Trisharnstoff)}

$0,5 \mathrm{ml} \mathrm{Sperma} \mathrm{wurde} \mathrm{mit} 4,5 \mathrm{ml} 3$ proz. $\mathrm{HClO}_{4}$ enteiweißt und filtriert. $1 \mathrm{ml}$ des Filtrats wurde mit $10 \mathrm{ml}$ Anthronreagenz $(500 \mathrm{mg}$ Anthron $+10 \mathrm{~g}$ Thioharnstoff in $1000 \mathrm{ml} 66$ proz. $\mathrm{H}_{2} \mathrm{SO}_{4}$ bei $80-90^{\circ} \mathrm{C}$ lösen) versetzt, $30 \mathrm{~min}$ stehen gelassen und die Extinktion gegen Wasser bei $1 \mathrm{~cm}$ Schichtdicke abgelesen. Der aus der Eichkurve erhaltene Wert muß mit 10 multipliziert werden. Angabe in $\mu \mathrm{g} / \mathrm{ml}$ Sperma. 
Tab. 1

Präzision und Richtigkeit von Standard-Fructose-Kontrollproben. Mittelwert aus je 10 Analysen. Sollwert: $2000 \mu \mathrm{g} / \mathrm{ml}$

\begin{tabular}{|c|c|c|c|c|c|}
\hline & Anthron & $\begin{array}{l}\text { Anthron }+ \\
\text { Thioharnstoff }\end{array}$ & Resorcin & Diphenylamin & $\begin{array}{l}\text { enzymatische } \\
\text { Methode }\end{array}$ \\
\hline$\overline{\mathrm{x}}(\mu \mathrm{g} / \mathrm{ml})$ & 2496 & 2260 & 2037 & 1966 & 2037 \\
\hline $\begin{array}{l}\text { Mittl. Abweichung des } \overline{\mathbf{x}} \text { vom } \\
\text { Sollwert in \% }\end{array}$ & $+24,8$ & +13 & $+1,9$ & $\pm 4,0$ & $+1,9$ \\
\hline Standardabweichung. $(\mu \mathrm{g} / \mathrm{ml})$ & \pm 148 & \pm 37 & \pm 43 & \pm 88 & \pm 54 \\
\hline Variationskoeffizient $\mathrm{V}(\%)$ & 5,92 & 1,63 & 2,11 & 4,5 & 2,65 \\
\hline Zulässige Streubreite $\bar{x} \pm 2 s$ & $2200-2792$ & $2186-2334$ & $1951-2123$ & $1790-2142$ & ' $1929-2145$ \\
\hline
\end{tabular}

\section{Enzymatische Fructose-Bestimmung}

Hierfür wurde das von BERNT und BERGMEYER (7) ausgearbeitete Verfahren benutzt. Für die Filtration nach Enteiweißung wurden Kieselgur-Filter verwendet.

\section{Ergebnisse}

Richtigkeit und Präzision von Standard-Kontrollproben

Zunächst wurde die zu erzielende Bestimmungsgenauigkeit der einzelnen Verfahren an einer Reihe von Fructose-Standardlösungen mit einem Sollwert von $2000 \mu \mathrm{g} / \mathrm{ml}$ untersucht.

Die Ergebnisse sind in Tabelle 1 aufgeführt und lassen exkennen, daß die schlechtesten Resultate mit dem Anthron- bzw. Anthron + Thioharnstoff-Nachweisreagenz exzielt wurden. Die übrigen 3 Verfahren sind hinsichtlich Präzision und Richtigkeit als gut zu bezeichnen. Als Ricbtigkeit (Kenngröße für systematische Fehler) wurde die Abwreichung des Mittelwertes $(\overline{\mathbf{x}})$ vom Sollwert der Kontrollprobe definiert.

Die Präzision, als Kenngröße für zufällige Fehler, wurde durch die Standard-Abweichung (s) ausgedrückt. Die reiative Standardabweichung (= Variationskoeffizient) wurde nach der allgemein bekannten Formel berechnet.

\section{Regressions-und Varianz-Analyse}

Aufgrund der vorangegangenen Untersuchungen konnten die beiden Anthron-Verfahren bei der statistischen Analyse der Sperma-Proben eliminiert werden.

\section{Regressions-Analyse und Korrelationskoeffizient}

Beim Vergleich der einzelnen Methoden miteinander resultierten die in Tabelle 2 aufgeführten Ergebnisse.

Tab. 2

Regressionsgeraden und Korrelationskoeffizienten für die durchgeführten Methoden-Vergleiche

\begin{tabular}{ccc}
\hline Methode & $\begin{array}{c}\text { GI. der Regressions- } \\
\text { geraden }\end{array}$ & $\begin{array}{c}\text { Korrelations: } \\
\text { koeffizient }\end{array}$ \\
\hline $\begin{array}{c}\text { Diphenylamin/ } \\
\text { Resorcin } \\
\begin{array}{c}\text { Diphenylamin/ } \\
\text { enzymatisch }\end{array}\end{array}$ & $\mathrm{y}=295+0,82 \times$ & $\mathrm{r}=+0,98$ \\
$\begin{array}{c}\text { Resorcin/ } \\
\text { enzymatisch }\end{array}$ & $\mathrm{y}=0,94 \mathrm{x}-\mathbf{7 0 , 0 9} \mathrm{x}-\mathbf{2 2 5 , 3}$ & $\mathrm{r}=+1,00$ \\
\hline
\end{tabular}

Aus Tabelle 2 und den Abbildungen 1-3 wird deutlich, $\mathrm{da} B$ eine lineare hochpositive Korrelation besteht, somit die genannten drei Verfahren praktisch vollständig übereinstimmen.

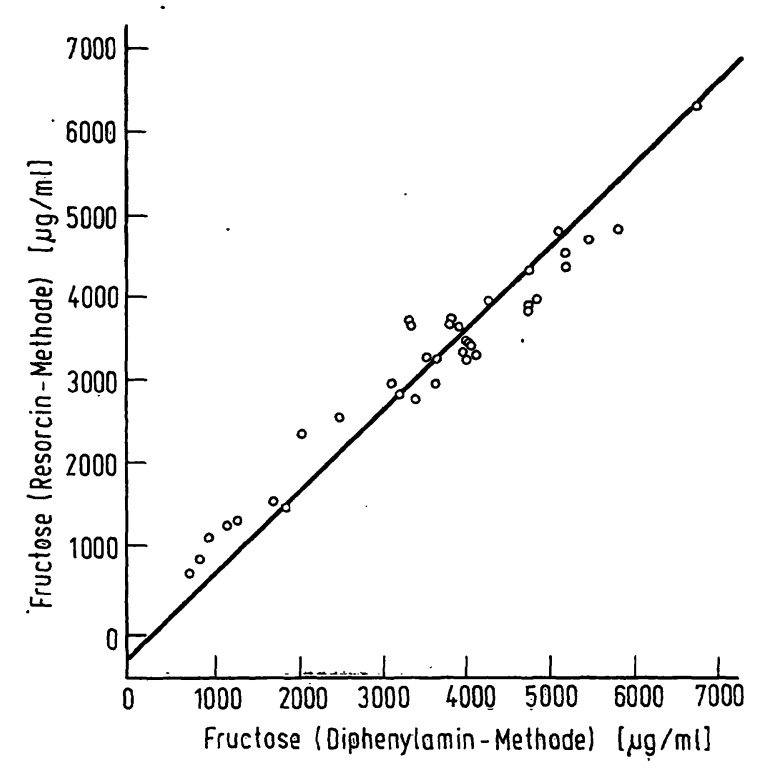

Abb. 1

Regressionslinie bei Vergleich von Diphenylamin- und Resorcin-

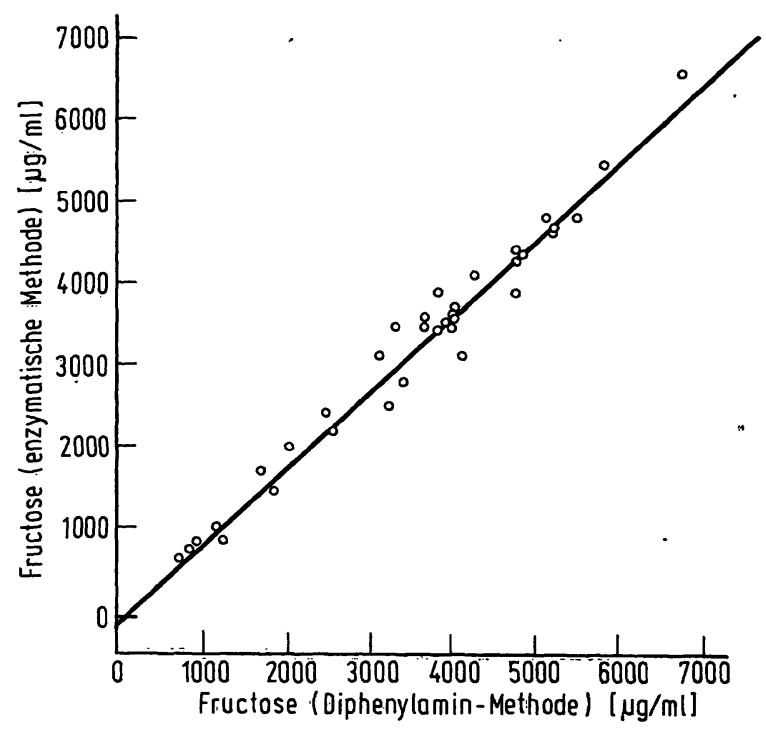

Abb. 2

Regressionslinie bei Vergleich von Diphenylamin- und enżymatischer Methode

\section{Varianzanalyse}

Die Prüfung der Regressionslinien wurde mit Hilfe des F-Testes durchgeführt.

Zunächst bestimmten wir die Summe der Ábweichungsquadrate insgesamt (SQ insgesamt) und dann die Varianz zwischen den Stichproben (SQ zwischen).

Die gewonnenen Werte sind in Tabelle 3 eingetragen. 


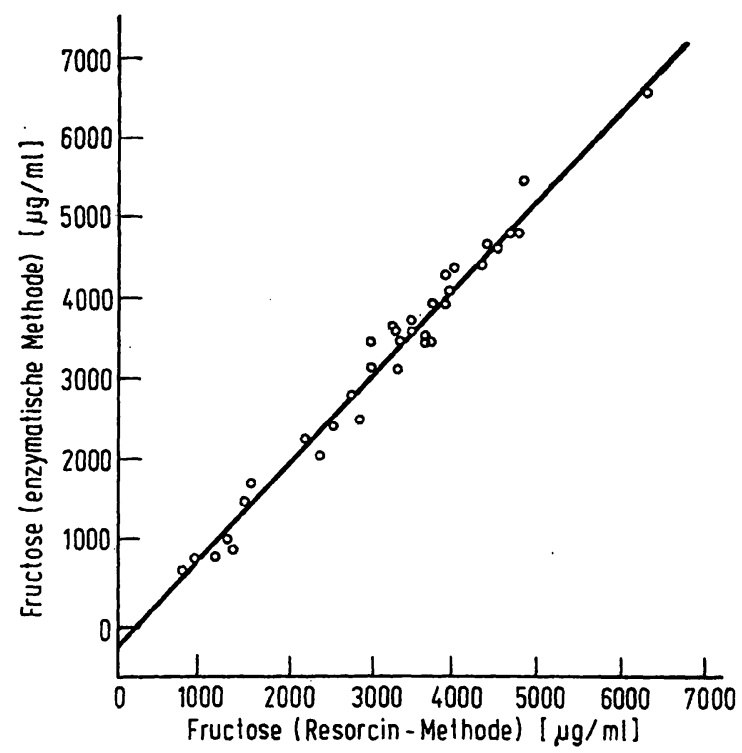

Regressionslinie bei Vergleich Mon $_{\text {Methode }}^{3}$ Resorcin- und enzymatischer

Tab. 3

Varianzanalytische Daten. $S Q(i g), \quad S Q(z w), S Q(i n)=$ Summe der Abweichungsquadrate insgesamt, zwischen den und innerhalb der Stichproben; $\mathbf{F G}=$ Freiheitsgrad

\begin{tabular}{lrrr}
\hline & SQ & FG & $\mathrm{S}^{2}=\frac{S Q}{S G}$ \\
\hline $\begin{array}{l}\text { Insgesamt (ig) } \\
\begin{array}{l}\text { Stichprobenfehler } \\
(\text { zw) }\end{array}\end{array}$ & 210036730 & 104 & \\
$\begin{array}{l}\text { Versuchsfehler (in) } \\
\text { (2504491 }\end{array}$ & 207532239 & 102 & 1252245,5 \\
\hline
\end{tabular}

Hieraus resultiert ein $Q-W e r t\left(Q=\frac{s^{2}(z w)}{s^{2}(i n)}\right)$ von 0,61 . $\mathrm{Da}$ dieser Wert wesentlich kleiner ist als der in den einschlägigen Tabellen, könnẹn die Abweichungen um die Regressionsgrade als zufällig angesehen werden. Die Regressionsgerade beschreibt also unser Zahlenmaterial ausreichend genau.

\section{Diskussion}

Aus dem methodischen Teil ist unschwer zu erkennen, daß die beiden Anthron-Methoden den relativ geringsten Arbeitsaufwand benötigen. Diesem offensichtlichen Vorteil steht eine hohe Ungenauigkeit hinsichtlich Präzision und Richtigkeit der Ergebnisse gegenüber. Dies zeigt sich in deutlicher Weise bei der Konstruktion der Eichkurve. Eine einmal aufgestellte Eichkurve war in aufeinander folgenden Tagen mit dem gleichen Reagenz nicht mehr reproduzierbar, d. h. die Steigung der Geraden schwankte von Tag zu Tag. Zum gleichen Ergebnis gelangten wir in LangzeitVersuchen, bei denen wir immer die gleiche FructoseKonzentration vorlegten und etwa 6 Wochen lang die Extinktion registrierten.

Daraufhin veränderten wir die Reaktionstemperatur, da diese einen wesentlichen Parameter darstellt. Wir stellten fest, daß die Werte am schlechtesten sind, wenn man nach der Originalvorschrift, d. h. ohne Kühlung, vorgeht. Arbeitet man mit Eiskühlung, so erhält man zwar etwas niedrigere Werte, die aber ebenfalls stark schwankten, d. h. zu hoch oder zu tief liegen, wie aus Tabelle 1 hervorgeht.

In diesem Zusammenhang wurde auch versucht, die Frage zu klären, bei welcher Temperatur (Raum-oder Kühlschrank-Tèmperatur von $+4^{\circ} \mathrm{C}$ ) das Anthronreagenz aufbewahrt werden soll. Eindeutige Unterschiede konnten wir hierbei nicht erkennen.

$\mathrm{Nach}$ unseren Erfahrungen muß festgestellt werden, daß die mit den genannten Anthron-Reagenzien aufgestellten Eichkurven für eine exakte Auswertung nicht verwendet werden können.

Zur Diphenylamin-Methode ist festzustellen, daß die Eichkurve gut reproduzierbar ist. Eine signifikante Änderung der Extinktionswerte tritt innerhalb von 3 Tagen nicht ein. Methodisch wurde die eingesetzte Spermamenge abgeändert. Bei Verwendung von $1 \mathrm{ml}$ Probe (statt 0,1 ml) werden die Extinktionen zu hoch. Dadurch ist eine exakte Ablesung nicht mehr gewährleistet.

Mit der Resorcin-Methode können nur dann gute und übereinstimmende Resultate erzielt werden, wenn das Nachweis-Reagenz täglich frisch hergestellt wird, denn mit zunehmendem Alter der Resorcinlösung flacht die Eichkurve ab. Wir konnten beobachten, daß z. B. für eine Konzentration von $100 \mu \mathrm{g} / \mathrm{ml}$ schon nach 2 Tagen die Extinktion von ursprünglich $0,7-0,6$ auf 0,35 , also fast $50 \%$, abfiel. In diesem Zusammenhang ist es völlig unverständlich, wie ScHIRreN (8) eine Tabelle angeben kann, in der Extinktionswerte entsprechenden Fructosewerten im Sperma zugeordnet werden.

Angesichts des oben genannten Sachverhalts ist eine derartige Empfehlung aufgrund der Streubreite, die nicht einmal angegeben wird, unzulässig. Außerdem ist der in 1. c. (8) auf S. 24 angegebene Multiplikationsfaktor unrichtig. Er müßte, gemäß den Angaben, nicht 80, sondern 44 lauten, wie die Úberprüfung ergeben hat. Der Faktor 80 hätte nur dann Gültigkeit, wenn die $0,1 \mathrm{ml}$ Sperma-Probe auf 4,0 $\mathrm{ml}$ (nicht 0,4 ml) mit dest. Wasser aufgefüllt wird.

Nach unseren Erfahrungen sollte auch für diese $\mathrm{Me}$ thode keine Eichkurve, sondern ein Standard verwendet werden. Der im methodischen Teil angegebene Faktor 2000 gilt nur, wenn die Konzentration des verwendeten Fructose-Standards $2000 \mu \mathrm{g} / \mathrm{ml}$ beträgt.

Das benutzte enzymatische Verfahren kann kommentarlos empfohlen werden. Bei Verwendung hinreichend reiner Enzyme und Coenzyme ist eine exakte Bestimmung immer gewährlcistet.

Abschließend sei die Rangfolge der benutzten Verfahren hinsichtlich Arbeitsaufwand angegeben:

Anthron

Anthron + Thioharnstoff

enzymatische Methode

zunehmender Aufwand

Resorcin

Diphenylamin

In bezug auf Präzision und Richtigkeit ergibt sich folgende Reihenfolge: 
enzymatische Analyse

Resorcin

Diphenylamin einander ebenbürtig

Anthron + Thioharnstoff \} einander ebenbürtig und Anthron
Zusammenfassend kann gesagt werden, daß bei allen Methoden die Berechnung der Analysenergebnisse unter Einbeziehung eines Standards der Auswertung über eine Eichkurve vorzuziehen ist. Nur dadurch ist es möglich, irrelevante Faktoren weitestgehend auszuschließen.

\section{Literatur}

1. MANN, TH. (1964), The biochemistry of semen and of the male reproductive tract, 1. Aufl., S. 237-265, Methuen \& Co, Ltd., London and John Wiley \& Sons, Inc., Neiv York. - 2. Hinsberg, K. \& Lang, K. (1957), Medizinische Chemie für den klinischen und theoretischen Gebrauch, 3. Aufl., S. 354-360, Urban \& Schwarzenberg, München-Berlin-Wien. - 3. Bonring, S. L. (1954), Arch. Biochem. Biophys. 52, 272-279. - 4. ROE, J. H.
(1955), J. Biol. Chem. 212, 335-343. - 5. MaRTIN, R. W. (1939), Hoppe-Seyler's Z. Physiol. Chem. 259, 62-74. - 6. RoE, J. H. (1934), J. Biol. Chem. 107, 15-22. - 7. Bernt, E. \& BergMEYER, H. U. (1970), Methoden det enzymatischen Analyse, Bd. 2, 2. Aufl., S. 1266-1269, Verlag Chemie Weinheim. 8. Schinren, C. (1971), Praktische Andrologie, 1. Aufl., S. 24-25, Verlag Brüder Hartmạnn, Berlin.

PD Dr. G. Peter

Biochem. Abteilung der Univ.-Hautklinik 8700 Würzburg

Josef-Schneider-Str. 2 